\title{
Efficient resummation of high post-Newtonian contributions to the binding energy
}

\author{
Stefano Foffa, ${ }^{a}$ Riccardo Sturani ${ }^{b}$ and William J. Torres Bobadilla ${ }^{c, d}$ \\ ${ }^{a}$ Département de Physique Théorique and Centre for Astroparticle Physics, Université de Genève, \\ CH-1211 Geneva, Switzerland \\ ${ }^{b}$ International Institute of Physics, Universidade Federal do Rio Grande do Norte, \\ Campus Universitário, Lagoa Nova, Natal-RN 59078-970, Brazil \\ ${ }^{c}$ Instituto de Física Corpuscular, Universitat de València, \\ Consejo Superior de Investigaciones Científicas, \\ Parc Cientific, E-46980 Paterna, Valencia, Spain \\ ${ }^{d}$ Max-Planck-Institut für Physik, Werner-Heisenberg-Institut, \\ 80805 München, Germany \\ E-mail: stefano.foffa@unige.ch, riccardo@iip.ufrn.br, \\ torres@mpp.mpg.de
}

ABstract: A factorisation property of Feynman diagrams in the context the Effective Field Theory approach to the compact binary problem has been recently employed to efficiently determine the static sector of the potential at fifth post-Newtonian (5PN) order. We extend this procedure to the case of non-static diagrams and we use it to fix, by means of elementary algebraic manipulations, the value of more than one thousand diagrams at $5 \mathrm{PN}$ order, that is a substantial fraction of the diagrams needed to fully determine the dynamics at $5 \mathrm{PN}$. This procedure addresses the redundancy problem that plagues the computation of the binding energy with respect to more "efficient" observables like the scattering angle, thus making the EFT approach in harmonic gauge at least as scalable as the others methods.

KEywords: Classical Theories of Gravity, Black Holes, Effective Field Theories

ARXIV EPRINT: 2010.13730 


\section{Contents}

1 Introduction 1

2 EFT and factorisation of non-static diagrams 3

3 Proof of concept 5

4 Conclusion $\quad 10$

A Detailed computation of a sample diagram 12

\section{Introduction}

The determination of the binding energy of spin-less compact binaries has recently reached the accuracy of the 4th order in the post-Newtonian (PN) approximation to General Relativity (GR), with several approaches obtaining the full 4PN contribution [1-13] with complete mutual agreement. The attention can now be focused on the next order, the 5PN level, and possibly beyond.

High-precision calculations are extremely important $[14,15]$ for maximising the physics output of the inspirals and coalescences of binary systems that are being and will be detected by the present and advanced versions of ground based interferometers [16, 17], as well as by third generation of detectors such as ET [18] and by the space detector LISA [19].

Remarkably, the interplay between modern techniques originated in high-energy physics with classical field theory methods has given further impulse to this quest, making the full determination of the 5PN sector a realistic goal for the near future. More in detail, the first post-Minkowskian part (i.e. the effective potential at first order in Newton constant $G$ and at all orders in the velocity, or 1PM for short) being known since long time [20], it has recently been re-derived [21, 22], and extended to 2PM [23]. Modern scattering amplitude techniques have been employed to determine the effective potential up to 3PM and at any order in the velocity expansion $[24,25]$ (not without some controversy in the interpretation of the results, see [26-29], confirming the necessity of multiple independent approaches to such difficult problems).

At the highest level of interaction at $5 \mathrm{PN}$ (that is $G^{6}$ ), the potential in the harmonic gauge has been determined using effective field theory (EFT)-based techniques within the Non-Relativistic-General-Relativity (NRGR) approach pioneered in [30], in close succession by two groups in [31] and [32]. The latter by a brute force calculation, the former exploiting a factorisation property that applies to all static diagrams at any odd-PN level. The extension of this factorisation property to non-static diagrams is the main focus of this work. 
More recently, a collaboration including the same authors as [32] has extended their results to cover some sectors of the $6 \mathrm{PN}$ order [33], while the contribution of hereditary effects to the conservative dynamics have been determined at 5PN in [34] along the same path established for the 4PN case within the EFT approach [35, 36].

In addition to the above mentioned efforts using EFT techniques, the combination of several techniques, spanning from PN and PM expansions, to self-force approach and effective-one-body re-summation has led to a systematisation of the center-of-mass Hamiltonian up to $6 \mathrm{PN}$, in which the still unknown sectors have been condensed into few unknown coefficients, respectively at $5 \mathrm{PN}$ (in the $G^{5}$ and $G^{6}$ sector) [37] and at $6 \mathrm{PN}$ (in the $G^{5}, G^{6}$ and $G^{7}$ sectors) [38-40]. This is a remarkable result, not quite for the small number of unknown coefficients, but rather for the insight gained by combining information coming from the computation of observables in different limits and within different approaches. A similar route has been followed in [41-43], where a formalism has been developed to map observables relative to bound states into unbound scatterings ones.

In particular, from the above analyses it has emerged that the scattering angle seems to store information about the two-body dynamics in a more efficient way than other observables. This can be seen in some remarkable properties of the scattering angle, like its simple dependence on the symmetric mass ratio [44], or the fact that it can be determined, at a given PM order, by a drastically lower number of diagrams with respect to the binding energy.

This means that, conversely, part of the information contained in the binding energy expression at a given PM or PN order is somehow redundant, as it should be determined by the knowledge of lower order contributions. This is exactly what has been enlightened in [31], where it is shown that static sectors at odd-PN orders are actually "redundant", in the sense that they are entirely composed by Feynman diagrams which are products of diagrams belonging to lower PN orders. Hence, we call such diagrams factorisable, which can be computed with simple algebraic operations, limiting the calculation of the nonfactorisable ones to the general, but computationally more demanding, method described in [10].

The paper is structured as follows. In section 2, we extend the identification and computation of factorisable diagrams to the general case (i.e. to non-static diagrams) and we provide a simple way of expressing factorisable diagrams in terms of lower order ones also in presence of time derivatives. We then apply this method to the $O\left(v^{2}\right)$ sector at $5 \mathrm{PN}$, in section 3 , and we determine its "redundant" contribution to the effective potential (that is, roughly two thirds of the diagrams involved in this sector) with a simple code which takes less than a minute on a normal laptop computer, ${ }^{1}$ as it involves only elementary algebraic manipulation. Finally, the implications of our work will be discussed in the conclusions section 4 .

\footnotetext{
${ }^{1}$ Considering that factorisable diagrams represent typically at least half of the total number, there is a sizable gain obtainable by a full-scale use of our method compared to the few hours of CPU time mentioned in [13] or the several weeks displayed in table 1 of [33].
} 


\section{EFT and factorisation of non-static diagrams}

The details of the procedure for computing the near-zone (i.e. at distances from the source smaller than the radiation wavelength) contribution to the effective potential have been outlined and discussed in several works [9-12, 31, 45, 46]. In this section, we recall just the points needed in this paper, starting from the fundamental path-integral equation,

$$
\exp \left[\mathrm{i} S_{\text {eff }}\left(x_{1}, x_{2}\right)\right]=\int \mathcal{D} g_{\mu \nu} \exp \left[\mathrm{i} S_{\text {bulk }}\left(g_{\mu \nu}\right)+\mathrm{i} S_{\mathrm{pp}}\left(g_{\mu \nu}, x_{1}, x_{2}\right)\right]
$$

with

$$
\begin{aligned}
S_{\text {bulk }} & =2 \Lambda^{2} \int \mathrm{d}^{d+1} x \sqrt{-g}\left[R(g)-\frac{1}{2} \Gamma^{\mu} \Gamma_{\mu}\right], \\
S_{\mathrm{pp}} & =-\sum_{a=1,2} m_{a} \int \mathrm{d} \tau_{a}=-\sum_{a=1,2} m_{a} \int \sqrt{-g_{\mu \nu}\left(x_{a}^{\mu}\right) \mathrm{d} x_{a}^{\mu} \mathrm{d} x_{a}^{\nu}},
\end{aligned}
$$

which relates the effective action to the fundamental actions describing Einstein gravity (plus the de-Donder gauge fixing term $-\frac{1}{2} \Gamma^{\mu} \Gamma_{\mu}$ ), minimally coupled to two spin-less point particles.

Working in the post-Newtonian framework, the above expression can be perturbatively evaluated in terms of Feynman diagrams, along the well-established formalism historically introduced in quantum field theory context and applied to the gravitationally bounded compact binary problem in [30], also known as NRGR. We adopt a Kaluza-Klein (KK) decomposition of the metric [47-49] into one scalar, one spatial vector, and one symmetric tensorial field, respectively called $\phi, A_{i}$ and $\sigma_{i j}$

$$
g_{\mu \nu}=e^{2 \phi / \Lambda}\left(\begin{array}{cc}
-1 & A_{j} / \Lambda \\
A_{i} / \Lambda & e^{-c_{d} \phi / \Lambda} \gamma_{i j}-A_{i} A_{j} / \Lambda^{2}
\end{array}\right)
$$

with $\gamma_{i j}=\delta_{i j}+\sigma_{i j} / \Lambda, c_{d}=2 \frac{(d-1)}{(d-2)}$ and $i, j$ running over the $d$ spatial dimensions.

The fundamental bulk action in (2.2) can be expressed in terms of the KK fields, but because of the purely algebraic method implemented in this work its explicit form is not needed here: our method computes factorisable diagrams by relying only on the knowledge of previously computed, lower order diagrams and world-line vertices.

As to $S_{\mathrm{pp}}$ it reads

$$
\begin{aligned}
S_{\mathrm{pp}} & =-\sum_{a=1,2} m_{a} \int \mathrm{d} \tau_{a} \\
& =-\sum_{a=1,2} m_{a} \int \mathrm{d} t_{a} e^{\phi / \Lambda} \sqrt{\left(1-\frac{A_{i}}{\Lambda} v_{a}^{i}\right)^{2}-e^{-c_{d} \phi / \Lambda}\left(v_{a}^{2}+\frac{\sigma_{i j}}{\Lambda} v_{a}^{i} v_{a}^{j}\right)}
\end{aligned}
$$

where $m_{a}$ the mass of $a$-th particle, and it can be used to derive the particle-gravity vertex interactions with different combinations of the KK fields, expanded up to the needed order in terms of the particle velocity $v$. E.g. world-line vertices involving respectively $n \phi, n \phi$ 
and one $A_{i}, n \phi$ and one $\sigma_{i j}$ fields bring the following factors from the expansion of eq. (2.5)

$$
\begin{aligned}
& \bar{\therefore} \stackrel{\wedge}{\therefore} \simeq-\frac{\mathrm{i} m}{n ! \Lambda^{n}}\left[1-\frac{d^{n}}{2(2-d)^{n}} v^{2}\right]
\end{aligned}
$$

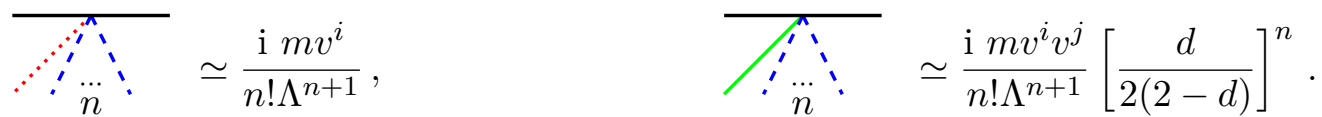

Black, dashed blue, dotted red, and green lines stand respectively for the particle worldline and the $\phi, A_{i}$ and $\sigma_{i j}$ propagators, while $\Lambda^{-2} \equiv 32 \pi G L^{d-3}$ is the $d$-dimensional gravitational coupling (we work in dimensional regularisation, with the arbitrary lengthscale $L$ eventually dropping out from observables).

To elucidate the notion of factorisable diagrams, let us consider a static 5PN diagram, together with its $p$-Fourier transformation, being $p$ the three-momentum exchanged between the two massive particles. In general, any gravity-amplitude of order $G_{N}^{\ell}$ can be mapped onto an $(\ell-1)$-loop 2-point function with massless internal lines and external momentum $p$. Then, after taking the $p$-Fourier transformation, one is led e.g. to,

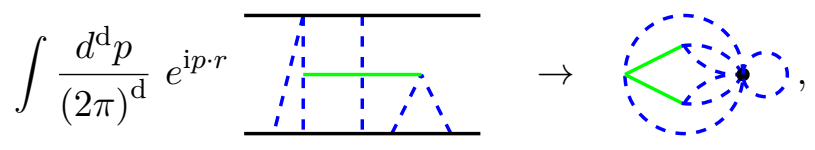

where in the final representation the external legs of the 1-point function have been joined and collapsed to a point to indicate the residual $r$ dependence of the diagram, finally represented by product of massless vacuum-vacuum diagrams.

As in [31], it has been observed that the value of the diagram on the left can be computed in terms of the values of the two sub-diagrams, in which it is naturally subdivided (above, on the right), according to a simple formula,

$$
\mathcal{V}^{\text {factorisable }}=\left(\mathcal{V}_{1} \times \mathcal{V}_{2}\right) \times \mathcal{K} \times \mathcal{C}
$$

with, $i) \mathcal{V}$ and $\mathcal{V}_{1,2}$ are, respectively, the values of the factorisable diagram and of the two sub-diagrams, ii) $\mathcal{K}$ accounts for the new matter interaction vertex of $\mathcal{V}$ (emerging from the sewing) out of matter interaction vertices of the two sub-diagrams and can be determined using equation (2.6), and iii) $\mathcal{C}=C^{\text {factorisable }} /\left(C_{1} \times C_{2}\right)$ where the $C^{\prime}$ 's are the combinatoric factors associated with each graph.

This procedure, introduced in [31], where it was used to obtain all the contributions to the static part of the 5PN effective action, can be extended to the non-static case with only minor adjustments, the main difference being that non-static diagrams can contain time derivatives which can "propagate" across factors a feature that can be naturally implemented in the codes usually employed to write the amplitude corresponding to a given graph. 

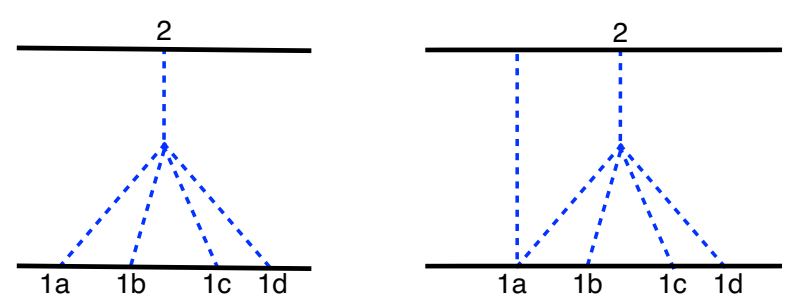

Figure 1. A 3-loop non-factorizable diagram (left) and a 4-loop factorizable one (right) which can be computed as the product of the former with the Newtonian one.

For instance, the 3-loop diagram in the left of figure 1 contains two times derivatives in the bulk $\phi^{5}$ vertex and has the following value,

$$
\begin{aligned}
\frac{G^{4} m_{1}^{4} m_{2}}{r^{4}}[ & \frac{8}{3} v_{1} \cdot v_{2}-\frac{4}{9} v_{1}^{2}+\frac{8}{3} v_{1}^{r 2}-\frac{16}{9} v_{1}^{r} v_{2}^{r} \\
& +\frac{2}{3}\left(4 v_{2}^{r}-3 v_{1}^{r}\right)\left(D_{1 a}+D_{1 b}+D_{1 c}+D_{1 d}\right)-\frac{8}{3} v_{1}^{r} D_{2} \\
& +\frac{2}{3}\left(2 D_{2}+D_{1 a}+D_{1 b}+D_{1 c}+D_{1 d}\right)\left(D_{1 a}+D_{1 b}+D_{1 c}+D_{1 d}\right) \\
& \left.-\frac{2}{3}\left(D_{1 a}^{2}+D_{1 b}^{2}+D_{1 c}^{2}+D_{1 d}^{2}\right)\right] .
\end{aligned}
$$

The symbols $D_{a i}$ represent time derivative operators acting on particle $a=1,2$ at worldline vertex $i$ on whatever sub-graph is eventually attached there (the index $i$ has been omitted when particle $a$ has only one vertex). By setting the $D_{a i}$ 's to zero, one obtains the value of the diagram which contributes to the $4 \mathrm{PN}$ effective action as computed in [11].

In this way, equation (2.8) holds also in the non-static case, provided the $\times$ symbol between $\mathcal{V}_{1}$ and $\mathcal{V}_{2}$ is interpreted in an extensive way to include also the action of the derivative operators $D_{a i}$ 's. For instance, if one wants to compute the value of the 4-loop diagram on the right part of figure 1, one has simply to "multiply" it by the value of the Newtonian diagram $N \equiv-\frac{G m_{1} m_{2}}{r}$, setting all the $D_{i}$ 's to zero except for $D_{1 a}$, which instead turns $N$ into $\dot{N}$. After inserting the appropriate factors $\mathcal{K}$ and $\mathcal{C}$ (see the appendix A for more details) one obtains the result,

$$
\frac{8}{9} \frac{G^{5} m_{1}^{4} m_{2}^{2}}{r^{5}}\left[2 v_{1}^{2}-12 v_{1} \cdot v_{2}-21 v_{1}^{r 2}+69 v_{1}^{r} v_{2}^{r}-12 v_{2}^{r 2}\right]
$$

without the need of performing any 4-loop momentum integration. A more involved example is discussed in detail in appendix A.

\section{Proof of concept}

In this section, we apply the method described above to the determination of factorisable diagrams up to order $v^{2}$ and up to $5 \mathrm{PN}$ (this procedure is generalisable to higher powers of $v$ and we leave this to future work). As a first step, we have modified our codes to include 
derivative operators, as in eq. (2.9), and we have recomputed all the non-factorisable Feynman diagrams containing up to two derivatives, and up to $4 \mathrm{PN}$, to take the $D_{i}$ operators into account. Second, we have used these prime diagrams as building blocks to generate all the factorisable ones. We have verified that we have been able in this way to reproduce the value of all the factorisable diagrams up to $4 \mathrm{PN}$, recovering the values already computed in $[11,45,50]$.

Next, we moved on to $5 \mathrm{PN}$, and we provide here a schematic summary of the result, where the $1220 G^{5} v^{2}$ factorisable diagrams (roughly two thirds of the total) have been divided into 8 subcategories. The diagram with a single $A_{i}$ propagator can be combined with four Newtonian diagrams, as schematically represented below

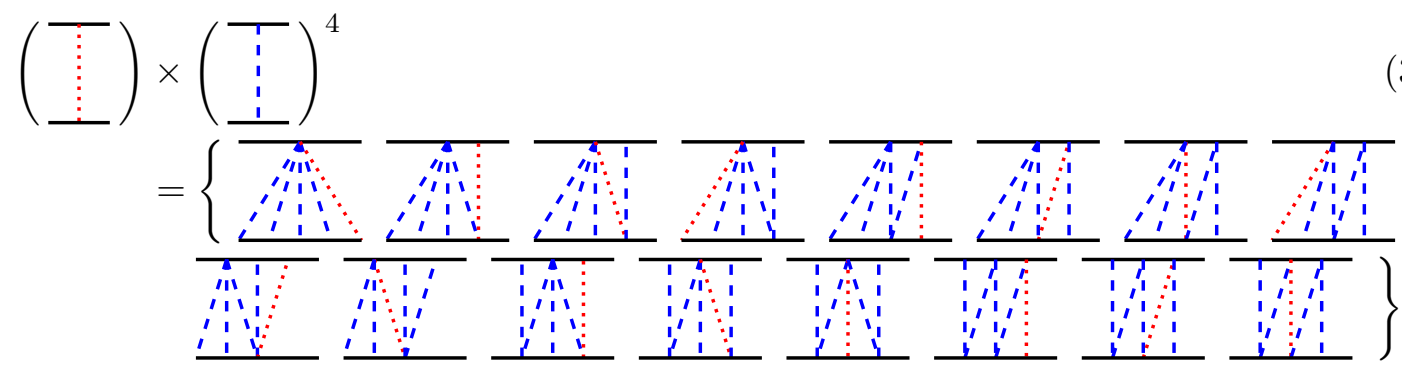

to give $16 G^{5} v^{2}$ factorisable diagrams, whose total contribution to the potential is

$$
V_{N^{4} G v^{2}}=\frac{1}{6} \frac{G^{5} m_{1}^{5} m_{2}}{r^{5}} v_{1} \cdot v_{2}+\frac{40}{3} \frac{G^{5} m_{1}^{4} m_{2}^{2}}{r^{5}} v_{1} \cdot v_{2}+\frac{45}{2} \frac{G^{5} m_{1}^{3} m_{2}^{3}}{r^{5}} v_{1} \cdot v_{2}+(1 \leftrightarrow 2) .
$$

The combination of three Newtonian diagrams with the $7 G^{2} v^{2}$ prime diagrams

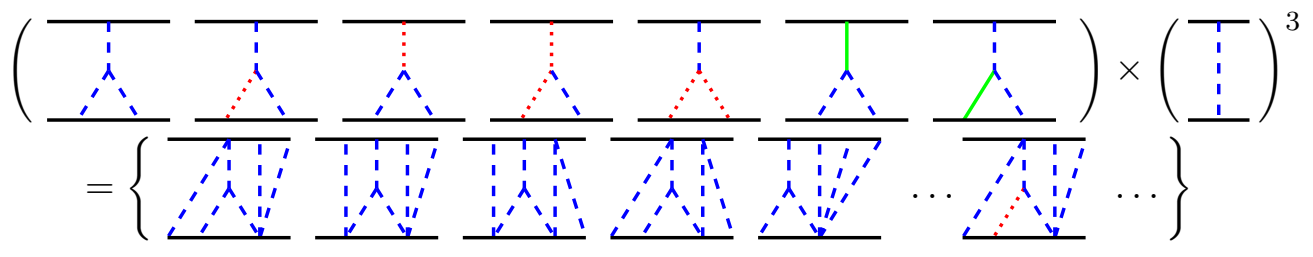

gives $135 G^{5} v^{2}$ factorisable diagrams, ${ }^{2}$ whose total contribution to the potential is

$$
\begin{aligned}
V_{N^{3} G^{2} v^{2}}= & \frac{G^{5} m_{1}^{5} m_{2}}{r^{5}}\left[\frac{7}{12} v^{2}+\frac{5}{3} v_{2}^{2}+\frac{1}{12} v^{r 2}-\frac{7}{3} v_{2}^{r 2}\right] \\
& +\frac{G^{5} m_{1}^{4} m_{2}^{2}}{r^{5}}\left[\frac{8 v^{r 2}}{\epsilon}+\frac{1}{3}\left(13 v_{1}^{2}-154 v_{1} \cdot v_{2}-17 v_{2}^{2}+335 v_{1}^{r 2}-478 v_{1}^{r} v_{2}^{r}+277 v_{2}^{r 2}\right)\right] \\
& +\frac{G^{5} m_{1}^{3} m_{2}^{3}}{r^{5}}\left[\frac{26 v_{1}^{r} v^{r}}{\epsilon}+\frac{1}{2}\left(49 v_{1}^{2}-147 v_{1} \cdot v_{2}+615 v_{1}^{r 2}-569 v_{1}^{r} v_{2}^{r}\right)\right]+(1 \leftrightarrow 2),
\end{aligned}
$$

with $\frac{1}{\epsilon} \equiv \frac{1}{(d-3)}-5 \log \left(\frac{r \sqrt{4 \pi \gamma_{E}}}{L}\right), v_{1,2}^{r} \equiv \frac{r \cdot v_{1,2}}{r}$, and $v \equiv v_{1}-v_{2}$.

\footnotetext{
${ }^{2}$ The dots in the second line in (3.3) stand for diagrams with the same topology as the first five with the replacement of the bulk triple vertex structure in the first line.
} 
The same $7 G^{2} v^{2}$ prime diagrams can also be combined with the 3 static prime $G^{3}$ diagrams

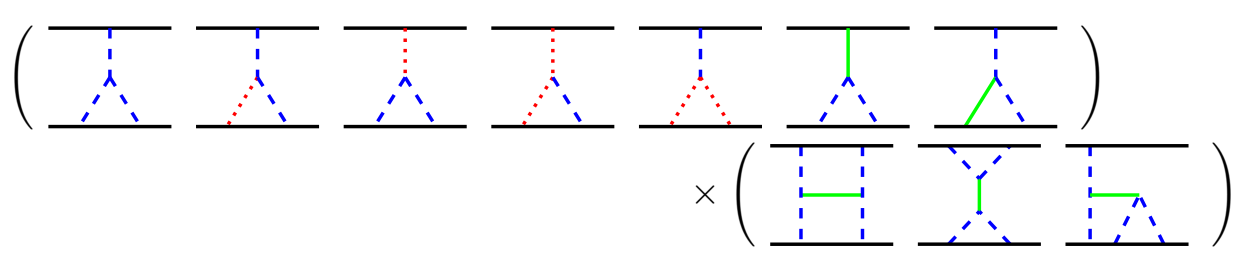

to give $85 G^{5} v^{2}$ factorisable diagrams (17 trivially vanishing because the central one in the second factor in eq. (3.5) is zero at leading order), whose total contribution to the potential is

$$
\begin{aligned}
V_{G^{3} v^{0} G^{2} v^{2}=} & \frac{G^{5} m_{1}^{5} m_{2}}{r^{5}}\left[\frac{7}{6} v^{2}-\frac{2}{3} v_{2}^{2}+\frac{1}{6} v^{r 2}-\frac{2}{3} v_{2}^{r 2}\right] \\
& +\frac{2 G^{5} m_{1}^{4} m_{2}^{2}}{3 r^{5}}\left[19 v_{1}^{2}-70 v_{1} \cdot v_{2}+7 v_{2}^{2}+65 v_{1}^{r 2}-82 v_{1}^{r} v_{2}^{r}+37 v_{2}^{r 2}\right] \\
& +\frac{G^{5} m_{1}^{3} m_{2}^{3}}{r^{5}}\left[-3 v_{1}^{2}-63 v_{1} \cdot v_{2}+215 v_{1}^{r 2}-153 v_{1}^{r} v_{2}^{r}\right]+(1 \leftrightarrow 2) .
\end{aligned}
$$

The combination of two Newtonian diagrams with the $28 G^{3} v^{2}$ prime diagrams

$$
\left(\begin{array}{c}
28 G^{3} v^{2} \\
\text { diagrams }
\end{array}\right) \times\left(\begin{array}{c}
T \\
\vdots \\
\vdots
\end{array}\right)^{2}
$$

gives $267 G^{5} v^{2}$ factorisable diagrams (11 of which trivially vanishing), whose total contribution to the potential is,

$$
\begin{aligned}
V_{N^{2} G^{3} v^{2}=\frac{G^{5} m_{1}^{5} m_{2}}{r^{5}}\left[\frac{1}{\epsilon}\right.}\left(\frac{41}{20} v_{1}^{2}-2 v_{1} \cdot v_{2}-\frac{203}{20} v_{1}^{r 2}+10 v_{1}^{r} v_{2}^{r}\right) \\
\left.+\left(\frac{3647}{600} v_{1}^{2}-\frac{19}{6} v_{1} \cdot v_{2}-\frac{27971}{600} v_{1}^{r 2}+\frac{287}{6} v_{1}^{r} v_{2}^{r}\right)\right] \\
+\frac{G^{5} m_{1}^{4} m_{2}^{2}}{r^{5}}\left[\frac{1}{\epsilon}\left(\frac{287}{10} v_{1}^{2}-28 v_{1} \cdot v_{2}-\frac{1191}{10} v_{1}^{r 2}+97 v_{1}^{r} v_{2}^{r}+20 v_{2}^{r 2}\right)\right. \\
+\left(\frac{37829}{300}-\frac{3}{2} \pi^{2}\right) v_{1}^{2}+\left(\frac{99}{32} \pi^{2}-\frac{178}{3}\right) v_{1} \cdot v_{2}+\left(41-\frac{3}{2} \pi^{2}\right) v_{2}^{2} \\
\left.+\left(\frac{9}{4} \pi^{2}-\frac{242297}{300}\right) v_{1}^{r 2}+\left(\frac{2792}{3}-\frac{129}{32} \pi^{2}\right) v_{1}^{r} v_{2}^{r}+\left(\frac{3}{2} \pi^{2}-167\right) v_{2}^{r 2}\right] \\
+\frac{G^{5} m_{1}^{3} m_{2}^{3}}{r^{5}}\left[\frac{1}{\epsilon}\left(\frac{369}{20} v_{1}^{2}-18 v_{1} \cdot v_{2}-\frac{927}{20} v_{1}^{r 2}+45 v_{1}^{r} v_{2}^{r}\right)\right. \\
+\left(\frac{43741}{200}-6 \pi^{2}\right) v_{1}^{2}+\left(\frac{99}{16} \pi^{2}-\frac{117}{2}\right) v_{1} \cdot v_{2} \\
\left.+\left(\frac{33}{4} \pi^{2}-\frac{227913}{200}\right) v_{1}^{r 2}+\left(\frac{2057}{2}-\frac{141}{16} \pi^{2}\right) v_{1}^{r} v_{2}^{r}\right]+(1 \leftrightarrow 2) .
\end{aligned}
$$


The product of the Newtonian diagram with the $171 G^{4} v^{2}$ prime diagrams

$$
\left(\begin{array}{c}
171 G^{4} v^{2} \\
\text { diagrams }
\end{array}\right) \times\left(\begin{array}{c}
T \\
\vdots \\
1
\end{array}\right)
$$

gives $665 G^{5} v^{2}$ factorisable diagrams (only 4 of which are trivially vanishing), whose total contribution to the potential is

$$
\begin{aligned}
& V_{N G^{4} v^{2}=} \frac{G^{5} m_{1}^{5} m_{2}}{r^{5}}\left[\frac{4}{\epsilon}\left(v_{1} \cdot v-5 v_{1}^{r} v^{r}\right)-\frac{2}{3}\left(v_{1}^{2}+5 v_{1} \cdot v_{2}-11 v_{1}^{r 2}+17 v_{1}^{r} v_{2}^{r}\right)\right] \\
&+\frac{G^{5} m_{1}^{4} m_{2}^{2}}{r^{5}}\left[\frac{4}{\epsilon}\left(-\frac{16}{15} v_{1}^{2}-\frac{2}{3} v_{1} \cdot v_{2}+2 v_{2}^{2}+\frac{47}{5} v_{1}^{r 2}-\frac{8}{3} v_{1}^{r} v_{2}^{r}-7 v_{2}^{r 2}\right)\right. \\
&-\left(\frac{29}{4} \pi^{2}+\frac{538}{75}\right) v_{1}^{2}+\left(\frac{7}{2} \pi^{2}-\frac{184}{3}\right) v_{1} \cdot v_{2}+\left(\frac{11}{4} \pi^{2}-\frac{134}{3}\right) v_{2}^{2} \\
&\left.+\left(\frac{781}{12} \pi^{2}+\frac{7216}{25}\right) v_{1}^{r 2}\left(\frac{451}{6} \pi^{2}+308\right) v_{1}^{r} v_{2}^{r}+\left(\frac{133}{12} \pi^{2}+\frac{212}{3}\right) v_{2}^{r 2}\right] \\
&+\frac{G^{5} m_{1}^{3} m_{2}^{3}}{r^{5}}\left[\frac{4}{\epsilon}\left(-\frac{34}{15} v_{1}^{2}+5 v_{1} \cdot v_{2}+\frac{419}{15} v_{1}^{r 2}-\frac{92}{3} v_{1}^{r} v_{2}^{r}\right)\right. \\
&-\left(\frac{7}{12} \pi^{2}+\frac{13012}{75}\right) v_{1}^{2}+\left(\frac{21}{4} \pi^{2}-72\right) v_{1} \cdot v_{2} \\
&+\left.\left(\frac{841}{12} \pi^{2}+\frac{24444}{25}\right) v_{1}^{r 2}-\left(\frac{299}{4} \pi^{2}+\frac{2312}{3}\right) v_{1}^{r} v_{2}^{r}\right]+(1 \leftrightarrow 2)
\end{aligned}
$$

The product of the Newtonian diagram, of the diagram with a single $A_{i}$ propagator, and of the three static prime $G^{3}$ diagrams

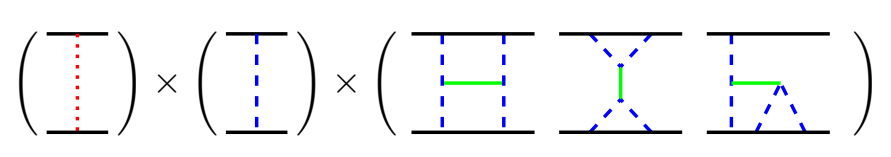

gives 27 factorisable diagrams ( 5 of which are trivially vanishing), whose total contribution to the potential is

$$
V_{N A G^{3} v^{0}}=\frac{4}{3} \frac{G^{5} m_{1}^{5} m_{2}}{r^{5}} v_{1} \cdot v_{2}+\frac{152}{3} \frac{G^{5} m_{1}^{4} m_{2}^{2}}{r^{5}} v_{1} \cdot v_{2}+76 \frac{G^{5} m_{1}^{3} m_{2}^{3}}{r^{5}} v_{1} \cdot v_{2}+(1 \leftrightarrow 2) .
$$

Finally, there are 25 diagrams which allow a non-vanishing static limit, thus contributing also at the 4PN dynamics (as computed in [10]). The static limit being just the leading order term of an expansion in the parameter $v^{2}$ (see the appendix for more details), the next-toleading order part of such diagrams contributes to the $5 \mathrm{PN}$ sector, and its evaluation can also be carried along the factorisation technique used so far. Hence, the 19 4PN diagrams resulting from the product of the three $G^{3} v^{0}$ with two Newtonian

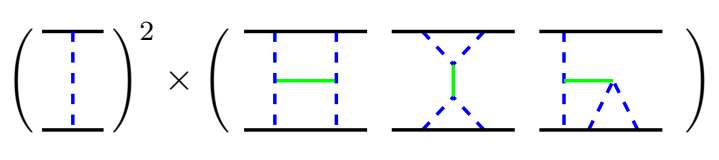


give, at next-to leading order in $v^{2}$

$$
\begin{aligned}
& V_{N^{2} G^{3} v^{0}}^{N L O}=\frac{G^{5} m_{1}^{5} m_{2}}{r^{5}}\left[\frac{1}{6 \epsilon}\left(v_{1} \cdot v_{2}-\frac{13}{10} v_{1}^{2}+\frac{59}{10} v_{1}^{r 2}-5 v_{1}^{r} v_{2}^{r}\right)-\frac{3691}{1800} v_{1}^{2}+\frac{17}{36} v_{1} \cdot v_{2}\right. \\
& \left.-\frac{9}{4} v_{2}^{2}+\frac{6113}{1800} v_{1}^{r 2}-\frac{115}{36} v_{1}^{r} v_{2}^{r}\right] \\
& +\frac{G^{5} m_{1}^{4} m_{2}^{2}}{r^{5}}\left[\frac{1}{3 \epsilon}\left(7 v_{1} \cdot v_{2}-\frac{91}{10} v_{1}^{2}+\frac{323}{10} v_{1}^{r 2}-26 v_{1}^{r} v_{2}^{r}\right)\right. \\
& +\left(\frac{7}{32} \pi^{2}-\frac{12329}{300}\right) v_{1}^{2}+\left(\frac{97}{18}-\frac{17}{32} \pi^{2}\right) v_{1} \cdot v_{2} \\
& +\left(\frac{7}{32} \pi^{2}-\frac{98}{9}\right) v_{2}^{2}+\left(\frac{26891}{900}-\frac{39}{32} \pi^{2}\right) v_{1}^{r 2} \\
& \left.+\left(\frac{63}{32} \pi^{2}-\frac{409}{18}\right) v_{1}^{r} v_{2}^{r}+\left(\frac{8}{3}-\frac{15}{32} \pi^{2}\right) v_{2}^{r 2}\right] \\
& +\frac{G^{5} m_{1}^{3} m_{2}^{3}}{r^{5}}\left[\frac{1}{\epsilon}\left(-\frac{39}{20} v_{1}^{2}+\frac{3}{2} v_{1} \cdot v_{2}+\frac{191}{60} v_{1}^{r 2}-\frac{11}{6} v_{1}^{r} v_{2}^{r}\right)+\left(\frac{7}{8} \pi^{2}-\frac{25669}{1800}\right) v_{1}^{2}\right. \\
& +\left(-\frac{17}{16} \pi^{2}+\frac{65}{36}\right) v_{1} \cdot v_{2}+\left(-\frac{53}{16} \pi^{2}+\frac{1713}{200}\right) v_{1}^{r 2} \\
& \left.+\left(\frac{31}{8} \pi^{2}+\frac{29}{4}\right) v_{1}^{r} v_{2}^{r}\right]+(1 \leftrightarrow 2) .
\end{aligned}
$$

To conclude, the $64 \mathrm{PN}$ diagrams resulting from the combination of five Newtonian diagrams

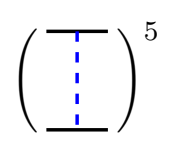

give, still at next-to-leading order in $v^{2}$

$$
\begin{aligned}
V_{N^{6}}^{N L O}= & \frac{G^{5} m_{1}^{5} m_{2}}{r^{5}}\left[-\frac{1}{16} v_{1}^{2}-\frac{1}{48} v_{1} \cdot v_{2}-\frac{81}{80} v_{2}^{2}-\frac{1}{12} v_{1}^{r 2}+\frac{5}{48} v_{1}^{r} v_{2}^{r}\right] \\
& +\frac{G^{5} m_{1}^{4} m_{2}^{2}}{r^{5}}\left[-\frac{5}{3} v_{1} \cdot v_{2}+2 v_{2}^{2}-3 v_{1}^{r 2}+\frac{13}{3} v_{1}^{r} v_{2}^{r}+\frac{1}{3} v_{2}^{r 2}\right] \\
& +\frac{G^{5} m_{1}^{3} m_{2}^{3}}{r^{5}}\left[-\frac{9}{8} v_{1}^{2}-\frac{45}{16} v_{1} \cdot v_{2}-\frac{11}{4} v_{1}^{r 2}+\frac{89}{16} v_{1}^{r} v_{2}^{r}\right]+(1 \leftrightarrow 2) .
\end{aligned}
$$

Summing all the above contributions, we obtain the total contributions of factorisable diagrams to the $G^{5} v^{2}$ sector of the $5 \mathrm{PN}$ potential

$$
\begin{aligned}
V_{v^{2}, \mathrm{fact}}^{5 P N}= & \frac{G^{5} m_{1}^{5} m_{2}}{r^{5}}\left[\frac{35}{6 \epsilon}\left(v_{1} \cdot v-5 v_{1}^{r} v^{r}\right)+\frac{727}{144} v_{1}^{2}-\frac{1159}{144} v_{1} \cdot v_{2}-\frac{41}{80} v_{2}^{2}\right. \\
& \left.-\frac{643}{18} v_{1}^{r 2}+\frac{4739}{144} v_{1}^{r} v_{2}^{r}-\frac{11}{4} v_{2}^{r 2}\right] \\
+ & \frac{G^{5} m_{1}^{4} m_{2}^{2}}{r^{5}}\left[\frac{1}{\epsilon}\left(\frac{107}{5} v_{1}^{2}-\frac{85}{3} v_{1} \cdot v_{2}+8 v_{2}^{2}-\frac{941}{15} v_{1}^{r 2}+\frac{185}{3} v_{1}^{r} v_{2}^{r}\right)\right. \\
& +\left(-\frac{273}{32} \pi^{2}+\frac{7112}{75}\right) v_{1}^{2}+\left(\frac{97}{16} \pi^{2}-\frac{2717}{18}\right) v_{1} \cdot v_{2}
\end{aligned}
$$



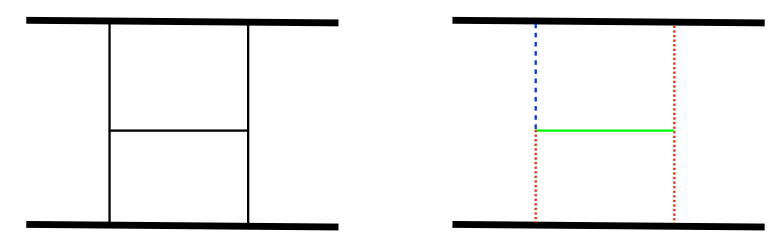

Figure 2. On the right, one of the hundreds of possible diagrams deriving from the 3PM topology on the left.

$$
\begin{aligned}
&+\left(\frac{47}{32} \pi^{2}-\frac{122}{9}\right) v_{2}^{2}+\left(\frac{6347}{96} \pi^{2}-\frac{75856}{225}\right) v_{1}^{r 2} \\
&+\left.\left(-\frac{3707}{48} \pi^{2}+\frac{7025}{18}\right) v_{1}^{r} v_{2}^{r}+\left(\frac{1163}{96} \pi^{2}+\frac{71}{3}\right) v_{2}^{r 2}\right] \\
&+\frac{G^{5} m_{1}^{3} m_{2}^{3}}{r^{5}}\left[\frac{1}{2 \epsilon}\left(\frac{223}{15} v_{1}^{2}+7 v_{1} \cdot v_{2}+\frac{2837}{15} v_{1}^{r 2}-211 v_{1}^{r} v_{2}^{r}\right)\right. \\
&+\left(\frac{92387}{1800}-\frac{137}{24} \pi^{2}\right) v_{1}^{2}+\left(\frac{83}{8} \pi^{2}-\frac{24409}{144}\right) v_{1} \cdot v_{2} \\
&\left.+\left(\frac{3601}{48} \pi^{2}+\frac{36651}{100}\right) v_{1}^{r 2}-\left(\frac{1275}{16} \pi^{2}+\frac{8009}{48}\right) v_{1}^{r} v_{2}^{r}\right] \\
&+(1 \leftrightarrow 2) .
\end{aligned}
$$

\section{Conclusion}

In the recent years, we have observed the emergence of novel ideas to provide precision calculations of the two-body dynamics at increasingly higher PN orders, even though to bootstrap the available techniques one needs to overcome three kinds of obstacles.

The first two main kinds of obstacle standing on the way of scaling precision computations of the two-body dynamics to higher order are intrinsically difficult master integrals (either in coordinate or in momentum space), and proliferation of terms. The former affect in equal measure all different methodologies employed so far, and the next hard step will be met in the high $G$ orders sectors at $6 \mathrm{PN}$.

On the contrary, the latter impacts unequally different observables and approaches. For instance, the EFT determination of Lagrangian effective potential involves a very large number of diagrams compared to other techniques, as gauge freedom, while providing several useful consistency checks, disperses information among several non-essential contributions. Furthermore, as noted throughout this paper, the explicit velocity counting, usually employed in the PN version of the EFT approach, involves a major proliferation of diagrams, only partially tampered by an efficient metric parametrisation, with respect to a full PM calculation. This is because several PN diagrams correspond to the same PM ones (also called topologies in our previous works), as depicted in figure 2 and this is the price to pay for dealing with simpler integrals with respect to full PM calculations. In effect, a reasonable price so far, considered the success of velocity-truncation approaches up to 4PN. 
The third obstacle is rooted in the observable itself, the binding energy which, as mentioned in the introduction, appears to be less efficient than the scattering angle in storing information. Therefore, several contributions at a given PN or PM order are actually simple algebraic functions of lower order ones, meaning that a good share of the diagram (or topology) proliferation could actually be overcome by more efficient bookkeeping algorithms. In view of this observation and former studies, we have explicitly exposed this mechanism by reducing the computation of all factorisable diagrams as algebraic functions of lower order ones. We have started with the static limit [31] and, in the present work, we have extended our formalism to the dynamic limit as well, thus paving the way to provide PN calculations in full generality.

The ratio of factorisable over non-factorisable diagrams or topologies is a generally growing function of the PN or PM order. The factorisable vs. non-factorisable diagram number ratio goes from 0.3 to 0.6 passing from $2 \mathrm{PN}$ to $4 \mathrm{PN} ;{ }^{3}$ considering diagrams with two powers of velocity, like the one computed in this paper, the ratio grows from 0.6 to 1.6 from $3 \mathrm{PN}$ to $5 \mathrm{PN}$, while in the static case the progression is from 2.75 to 6.5 , thus suggesting a growing trend of this ratio, implying a higher and higher relative weight of factorisable diagrams with respect to non-factorisable ones, at higher powers of $G$. Counting the number of topologies instead of diagrams, there are 118 factorisable topologies contributing at 5PN, vs. 62 non-factorisable ones. These numbers indicate that any strategy or approach to scale perturbative calculations at higher orders will benefit from optimisation procedures like the one put forward in this work.

The work [51] appeared online on the arXiv on the same day of this paper, containing the result of the full near zone computation of the fifth post-Newtonian order two-body dynamics. We would like to point out that the results presented in this paper, corresponding to the factorisable diagrams, have been explicitly verified via private communications with the authors of [51], finding complete agreement with their corresponding subset of diagrams.

\section{Acknowledgments}

We wish to thank Pierpaolo Mastrolia and Christian Sturm for countless discussions and unwavering support during the completion of this work. We thank the authors of [51] for helping us correct eq. (3.10) in the first arXiv version of this paper. S.F. is supported by the Fonds National Suisse and by the SwissMap NCCR. R.S. is partially supported by CNPq. W.J.T. has been supported in part by Grants No. FPA2017-84445-P, SEV-20140398 (AEI/ERDF, EU), the COST Action CA16201 PARTICLEFACE and the "Juan de la Cierva Formación" program (FJCI-2017-32128).

\footnotetext{
${ }^{3}$ The comparison must be made between PN or PM orders with the same parity.
} 


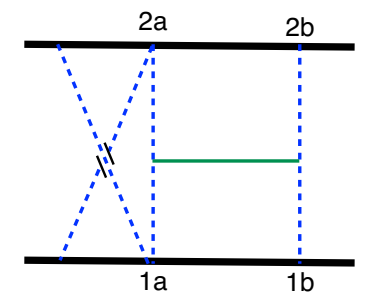

Figure 3. Representative diagram at $4 \mathrm{PN}$.

\section{A Detailed computation of a sample diagram}

In this appendix, we explicitly show how the procedure described in section 2 works in detail on the diagram of figure 3 .

The diagram of figure 3 is the product of an H-shaped diagram with two Newtonian ones (one attached at the upper left vertex, another attached at the lower left one). This belong to the most difficult category, because all factors have to be evaluated at next-toleading order in time derivatives, meaning that derivative operators act in every direction (from the H-shaped to the Newtonians, and vice-versa). The majority of the other diagrams have derivative operators, when present at all, acting only in one direction, from the higherloop factor towards the Newtonians.

We can write each of the Newtonian building blocks as

$$
N=N_{0}+N_{v^{2}}
$$

where

$$
\begin{aligned}
N_{0} & =-\frac{G m_{1} m_{2}}{r}\left[1-(d-3)\left(\log r-\frac{1}{2}\right)\right], \\
N_{v^{2}} & =-\frac{G m_{1} m_{2}}{2 r}\left[3 v_{1}^{2}+3 v_{2}^{2}+v_{1} \cdot v_{2}-v_{1}^{r} v_{2}^{r}-r D_{2} v_{1}^{r}+r D_{1} v_{2}-r^{2} D_{1} D_{2}\right],
\end{aligned}
$$

and we are setting here $L=\sqrt{4 \pi \gamma_{E}}$ in order to simplify notations. The $N_{v^{2}}$ term, that is the next-to-leading term of the Newtonian diagram in the velocity expansion, comes from the expansion of the propagator around $k_{0} \sim 0$, e.g. for the $\phi$ propagator $P_{\phi}$ :

$$
\begin{aligned}
P_{\phi}\left[t-t^{\prime}, x(t)-x^{\prime}\left(t^{\prime}\right)\right] & =-\frac{1}{2 c_{d}} \int \frac{d \omega}{2 \pi} \frac{d^{\mathrm{d}} k}{(2 \pi)^{\mathrm{d}}} \frac{e^{i k_{0}\left(t-t^{\prime}\right)-i k \cdot\left[x(t)-x^{\prime}\left(t^{\prime}\right)\right]}}{k^{2}-k_{0}^{2}} \\
& \simeq \int \frac{d^{\mathrm{d}} k}{(2 \pi)^{\mathrm{d}}} \frac{e^{-i k \cdot\left[x(t)-x^{\prime}\left(t^{\prime}\right)\right]}}{k^{2}}\left(1+\frac{\partial_{t} \partial_{t^{\prime}}}{k^{2}}+\ldots\right) \delta\left(t-t^{\prime}\right),
\end{aligned}
$$

which generate velocity terms when the time derivatives act on the particle worldlines present in the exponential factor.

We will also need the derivative of the static term (the derivative of $N_{v^{2}}$ would obviously be $\mathcal{O}\left(v^{2}\right) \times N_{v^{2}}$, therefore it is not needed here):

$$
\dot{N}_{0}=\frac{G m_{1} m_{2}}{r^{2}} v^{r}\left[1-(d-3)\left(\log r-\frac{3}{2}\right)\right] .
$$


As to the H-shaped diagram, it reads

$$
F_{3}=\frac{G^{3} m_{1}^{2} m_{2}^{2}}{r^{3}}\left[f_{0}+f_{v^{2}}+r f_{i} D_{i}+r^{2} f_{i j} D_{i} D_{j}\right]
$$

with $i, j=1 a, 2 a, 1 b, 2 b$ and $f_{0}=-1$. As to $f_{v^{2}}, f_{i}, f_{i j}$, they are velocity-dependent terms generated by the same propagator expansion described above, as well as by a time dependent part contained in the $\phi^{2} \sigma$ bulk interaction vertex. They read

$$
\begin{aligned}
f_{v^{2}} & =-\frac{161}{36}\left(v_{1}^{2}+v_{2}^{2}\right)+\frac{25}{36} v_{1} \cdot v_{2}+\frac{29}{12}\left(v_{1}^{r 2}+v_{2}^{r 2}\right)-\frac{49}{12} v_{1}^{r} v_{2}^{r}+\frac{\pi^{2}}{16}\left(v^{2}-3 v^{r 2}\right) \\
f_{1 a} & =\left(\frac{4}{9} v_{1}^{r}+\frac{11}{36} v_{2}^{r}-\frac{\pi^{2}}{16} v_{2}^{r}\right) \\
f_{2 a} & =-\left(\frac{4}{9} v_{2}^{r}+\frac{11}{36} v_{1}^{r}-\frac{\pi^{2}}{16} v_{1}^{r}\right) \\
f_{1 a, 2 a} & =\left(-\frac{1}{3(d-3)}+\log r-\frac{29}{18}+\frac{\pi^{2}}{8}\right),
\end{aligned}
$$

while we can ignore the other $f_{i}$ 's because the corresponding operators do not act on anything. For the same reason, we can also set $D_{1}=0$ in the Newtonian sub-diagram attached to the upper vertex, and analogously $D_{2}=0$ for the other one. We also observe that derivative operators of each factor act just on the static part of the other factors (again, because we are working at $\mathcal{O}\left(v^{2}\right)$ ) so, for the sake of computing the generalised product $N \times N \times F_{3}$, we can make the following replacements:

$$
\begin{gathered}
D_{1}[\text { lower Newtonian factor }] \rightarrow-4 \frac{v_{r}}{r}, \\
D_{2}[\text { upper Newtonian factor }] \rightarrow-4 \frac{v_{r}}{r},
\end{gathered}
$$

and

$$
D_{1 a}, D_{2 a}[\text { H diagram }] \rightarrow \frac{\dot{N}_{0}}{N_{0}}
$$

This brings to

$$
\begin{array}{r}
N \times N \times F_{3}=\frac{G^{3} m_{1}^{2} m_{2}^{2}}{r^{3}}\left(N_{0}+\left.N_{v^{2}}\right|_{D_{1}=-4 \frac{v^{r}}{r}, D_{2}=0}\right)\left(N_{0}+\left.N_{v^{2}}\right|_{D_{2}=-4 \frac{v^{r}}{r}, D_{1}=0}\right) \\
\times\left[-1+f_{v^{2}}+r f_{1 a} \frac{\dot{N}_{0}}{N_{0}}+r f_{2 a} \frac{\dot{N}_{0}}{N_{0}}+r^{2} f_{1 a, 2 a}\left(\frac{\dot{N}_{0}}{N_{0}}\right)^{2}\right] \\
\simeq \frac{G^{3} m_{1}^{2} m_{2}^{2}}{r^{3}}\left[N_{0}^{2}\left(-1+f_{v^{2}}\right)+N_{0} \dot{N}_{0}\left(r f_{1 a}+r f_{2 a}\right)\right. \\
\left.-N_{0}\left(\left.N_{v^{2}}\right|_{D_{2}=0} ^{D_{1}=-4 \frac{v^{r}}{r}}+\left.N_{v^{2}}\right|_{D_{2}=-4 \frac{v^{r}}{r}} ^{D_{1}=0}\right)+r^{2} f_{1 a, 2 a}\left(\dot{N}_{0}\right)^{2}\right] .
\end{array}
$$


Since the only pole is in $f_{1 a, 2 a}$, we can trade $N_{0}$ and its derivative for their three dimensional value everywhere except in the last term, where we have to keep the full $\mathcal{O}(d-3)$ expressions,

$$
\begin{aligned}
N \times N \times F_{3} \simeq \frac{G^{5} m_{1}^{4} m_{2}^{4}}{r^{5}}\{ & -1+f_{v^{2}}-\frac{v^{r}}{r}\left(r f_{1 a}+r f_{2 a}\right) \\
& -\left(3 v_{1}^{2}+3 v_{2}^{2}+v_{1} \cdot v_{2}-v_{1}^{r} v_{2}^{r}+2 v_{1}^{r} v^{r}-2 v_{2}^{r} v^{r}\right) \\
& \left.+r^{2} f_{1 a, 2 a}\left(\frac{v^{r}}{r}\right)^{2}\left[1-2(d-3)\left(\log r-\frac{3}{2}\right)\right]\right\} \\
\simeq \frac{G^{5} m_{1}^{4} m_{2}^{4}}{r^{5}} & \left\{-1+f_{v^{2}}-\left(\frac{5}{36}+\frac{\pi^{2}}{16}\right) v^{r 2}\right. \\
& -\left(3 v_{1}^{2}+3 v_{2}^{2}+v_{1} \cdot v_{2}+2 v_{1}^{r 2}+2 v_{2}^{r 2}-5 v_{1}^{r} v_{2}^{r}\right) \\
& \left.+v^{r 2}\left[-\frac{1}{3(d-3)}+\log r-\frac{29}{18}+\frac{\pi^{2}}{8}+\frac{2}{3}\left(\log r-\frac{3}{2}\right)+\mathcal{O}(\epsilon)\right]\right\} \\
\simeq \frac{G^{5} m_{1}^{4} m_{2}^{4}}{r^{5}} & {\left[-\frac{v^{r 2}}{3 \epsilon}-1-\frac{269}{36}\left(v_{1}^{2}+v_{2}^{2}\right)-\frac{11}{36} v_{1} \cdot v_{2}\right.} \\
& \left.-\frac{7}{3}\left(v_{1}^{r 2}+v_{2}^{r 2}\right)+\frac{77}{12} v_{1}^{r} v_{2}^{r}+\frac{\pi^{2}}{16}\left(v^{2}-2 v^{r 2}\right)\right] .
\end{aligned}
$$

Next, we have to multiply by the symmetry factor and by the vertex corrections up to $O\left(v^{2}\right)$ :

$$
\begin{aligned}
C^{\text {factorisable }} \times\left(\frac{V_{\phi^{2}}}{V_{\phi}^{2}}\right)_{1} \times\left(\frac{V_{\phi^{2}}}{V_{\phi}^{2}}\right)_{2} & =8 \frac{1-\frac{d^{2}}{2(d-2)^{2}} v_{1}^{2}}{2 m_{1}\left(1+\frac{d}{2(d-2)} v_{1}^{2}\right)^{2}} \frac{1-\frac{d^{2}}{2(d-2)^{2}} v_{2}^{2}}{2 m_{2}\left(1+\frac{d}{2(d-2)} v_{2}^{2}\right)^{2}} \\
& \simeq \frac{2}{m_{1} m_{2}}\left[1-\frac{3 d(2 d-4)}{2(d-2)^{2}}\left(v_{1}^{2}+v_{2}^{2}\right)\right] \\
& =\frac{2}{m_{1} m_{2}}\left[1-\frac{15}{2}\left(v_{1}^{2}+v_{2}^{2}\right)+\mathcal{O}(d-3)\right]
\end{aligned}
$$

and we can neglect the $\mathcal{O}(d-3)$ part because the pole in the previous expression is already $\mathcal{O}\left(v^{2}\right)$. Hence, the final result gives

$$
\begin{array}{r}
N \times N \times F_{3}=\frac{2 G^{5} m_{1}^{3} m_{2}^{3}}{r^{5}}\left[-\frac{v^{r 2}}{3 \epsilon}-1+\frac{1}{36}\left(v_{1}^{2}+v_{2}^{2}\right)-\frac{11}{36} v_{1} \cdot v_{2}\right. \\
\left.-\frac{7}{3}\left(v_{1}^{r 2}+v_{2}^{r 2}\right)+\frac{77}{12} v_{1}^{r} v_{2}^{r}+\frac{\pi^{2}}{16}\left(v^{2}-2 v^{r 2}\right)\right] .
\end{array}
$$

The operations described in this appendix were straightforwardly automated and adapted to all the 1220 factorisable diagram (the case displayed here is the most involved one), thus reducing the evaluation of all of them to a few minutes run.

Open Access. This article is distributed under the terms of the Creative Commons Attribution License (CC-BY 4.0), which permits any use, distribution and reproduction in any medium, provided the original author(s) and source are credited. 


\section{References}

[1] T. Damour, P. Jaranowski and G. Schäfer, Nonlocal-in-time action for the fourth post-Newtonian conservative dynamics of two-body systems, Phys. Rev. D 89 (2014) 064058 [arXiv:1401.4548] [INSPIRE].

[2] T. Damour, P. Jaranowski and G. Schäfer, Fourth post-Newtonian effective one-body dynamics, Phys. Rev. D 91 (2015) 084024 [arXiv: 1502.07245] [InSPIRE].

[3] T. Damour, P. Jaranowski and G. Schäfer, Conservative dynamics of two-body systems at the fourth post-Newtonian approximation of general relativity, Phys. Rev. D 93 (2016) 084014 [arXiv: 1601.01283] [INSPIRE].

[4] L. Bernard, L. Blanchet, A. Bohé, G. Faye and S. Marsat, Fokker action of nonspinning compact binaries at the fourth post-Newtonian approximation, Phys. Rev. D 93 (2016) 084037 [arXiv: 1512.02876] [INSPIRE].

[5] L. Bernard, L. Blanchet, A. Bohé, G. Faye and S. Marsat, Energy and periastron advance of compact binaries on circular orbits at the fourth post-Newtonian order, Phys. Rev. D 95 (2017) 044026 [arXiv:1610.07934] [INSPIRE].

[6] L. Bernard, L. Blanchet, A. Bohé, G. Faye and S. Marsat, Dimensional regularization of the IR divergences in the Fokker action of point-particle binaries at the fourth post-Newtonian order, Phys. Rev. D 96 (2017) 104043 [arXiv:1706.08480] [InSPIRE].

[7] T. Marchand, L. Bernard, L. Blanchet and G. Faye, Ambiguity-Free Completion of the Equations of Motion of Compact Binary Systems at the Fourth Post-Newtonian Order, Phys. Rev. D 97 (2018) 044023 [arXiv: 1707.09289] [INSPIRE].

[8] L. Bernard, L. Blanchet, G. Faye and T. Marchand, Center-of-Mass Equations of Motion and Conserved Integrals of Compact Binary Systems at the Fourth Post-Newtonian Order, Phys. Rev. D 97 (2018) 044037 [arXiv:1711.00283] [InSPIRE].

[9] S. Foffa and R. Sturani, Dynamics of the gravitational two-body problem at fourth post-Newtonian order and at quadratic order in the Newton constant, Phys. Rev. D 87 (2013) 064011 [arXiv: 1206.7087] [INSPIRE].

[10] S. Foffa, P. Mastrolia, R. Sturani and C. Sturm, Effective field theory approach to the gravitational two-body dynamics, at fourth post-Newtonian order and quintic in the Newton constant, Phys. Rev. D 95 (2017) 104009 [arXiv:1612.00482] [InSPIRE].

[11] S. Foffa and R. Sturani, Conservative dynamics of binary systems to fourth Post-Newtonian order in the EFT approach I: Regularized Lagrangian, Phys. Rev. D 100 (2019) 024047 [arXiv: 1903.05113] [INSPIRE].

[12] S. Foffa, R.A. Porto, I. Rothstein and R. Sturani, Conservative dynamics of binary systems to fourth Post-Newtonian order in the EFT approach II: Renormalized Lagrangian, Phys. Rev. D 100 (2019) 024048 [arXiv: 1903.05118] [INSPIRE].

[13] J. Blümlein, A. Maier, P. Marquard and G. Schäfer, Fourth post-Newtonian Hamiltonian dynamics of two-body systems from an effective field theory approach, Nucl. Phys. B 955 (2020) 115041 [arXiv:2003.01692] [INSPIRE].

[14] L. Lindblom, B.J. Owen and D.A. Brown, Model Waveform Accuracy Standards for Gravitational Wave Data Analysis, Phys. Rev. D 78 (2008) 124020 [arXiv:0809.3844] [INSPIRE]. 
[15] A. Antonelli, A. Buonanno, J. Steinhoff, M. van de Meent and J. Vines, Energetics of two-body Hamiltonians in post-Minkowskian gravity, Phys. Rev. D 99 (2019) 104004 [arXiv: 1901.07102] [INSPIRE].

[16] LIGO Scientific collaboration, Advanced LIGO, Class. Quant. Grav. 32 (2015) 074001 [arXiv: 1411.4547] [INSPIRE].

[17] VIRGO collaboration, Advanced Virgo: a second-generation interferometric gravitational wave detector, Class. Quant. Grav. 32 (2015) 024001 [arXiv: 1408.3978] [INSPIRE].

[18] M. Punturo et al., The Einstein Telescope: A third-generation gravitational wave observatory, Class. Quant. Grav. 27 (2010) 194002 [INSPIRE].

[19] LISA collaboration, Laser Interferometer Space Antenna, arXiv:1702.00786 [INSPIRE].

[20] T. Ledvinka, G. Schaefer and J. Bicak, Relativistic Closed-Form Hamiltonian for Many-Body Gravitating Systems in the Post-Minkowskian Approximation, Phys. Rev. Lett. 100 (2008) 251101 [arXiv: 0807.0214] [INSPIRE].

[21] L. Blanchet and A.S. Fokas, Equations of motion of self-gravitating $N$-body systems in the first post-Minkowskian approximation, Phys. Rev. D 98 (2018) 084005 [arXiv:1806.08347] [INSPIRE].

[22] S. Foffa, Gravitating binaries at 5PN in the post-Minkowskian approximation, Phys. Rev. D 89 (2014) 024019 [arXiv: 1309.3956] [INSPIRE].

[23] T. Damour, High-energy gravitational scattering and the general relativistic two-body problem, Phys. Rev. D 97 (2018) 044038 [arXiv:1710.10599] [INSPIRE].

[24] C. Cheung, I.Z. Rothstein and M.P. Solon, From Scattering Amplitudes to Classical Potentials in the Post-Minkowskian Expansion, Phys. Rev. Lett. 121 (2018) 251101 [arXiv: 1808.02489] [INSPIRE].

[25] Z. Bern, C. Cheung, R. Roiban, C.-H. Shen, M.P. Solon and M. Zeng, Scattering Amplitudes and the Conservative Hamiltonian for Binary Systems at Third Post-Minkowskian Order, Phys. Rev. Lett. 122 (2019) 201603 [arXiv:1901.04424] [INSPIRE].

[26] T. Damour, Classical and quantum scattering in post-Minkowskian gravity, Phys. Rev. D 102 (2020) 024060 [arXiv: 1912.02139] [INSPIRE].

[27] Z. Bern, H. Ita, J. Parra-Martinez and M.S. Ruf, Universality in the classical limit of massless gravitational scattering, Phys. Rev. Lett. 125 (2020) 031601 [arXiv:2002.02459] [INSPIRE].

[28] P. Di Vecchia, C. Heissenberg, R. Russo and G. Veneziano, Universality of ultra-relativistic gravitational scattering, Phys. Lett. B 811 (2020) 135924 [arXiv:2008.12743] [INSPIRE].

[29] T. Damour, Radiative contribution to classical gravitational scattering at the third order in G, Phys. Rev. D 102 (2020) 124008 [arXiv:2010.01641] [inSPIRE].

[30] W.D. Goldberger and I.Z. Rothstein, An Effective field theory of gravity for extended objects, Phys. Rev. D 73 (2006) 104029 [hep-th/0409156] [INSPIRE].

[31] S. Foffa, P. Mastrolia, R. Sturani, C. Sturm and W.J. Torres Bobadilla, Static two-body potential at fifth post-Newtonian order, Phys. Rev. Lett. 122 (2019) 241605 [arXiv: 1902.10571] [INSPIRE].

[32] J. Blümlein, A. Maier and P. Marquard, Five-Loop Static Contribution to the Gravitational Interaction Potential of Two Point Masses, Phys. Lett. B 800 (2020) 135100 [arXiv: 1902.11180] [INSPIRE]. 
[33] J. Blümlein, A. Maier, P. Marquard and G. Schäfer, Testing binary dynamics in gravity at the sixth post-Newtonian level, Phys. Lett. B 807 (2020) 135496 [arXiv: 2003.07145] [InSPIRE].

[34] S. Foffa and R. Sturani, Hereditary terms at next-to-leading order in two-body gravitational dynamics, Phys. Rev. D 101 (2020) 064033 [arXiv:1907.02869] [InSPIRE].

[35] S. Foffa and R. Sturani, Tail terms in gravitational radiation reaction via effective field theory, Phys. Rev. D 87 (2013) 044056 [arXiv:1111.5488] [INSPIRE].

[36] R.A. Porto and I.Z. Rothstein, Apparent ambiguities in the post-Newtonian expansion for binary systems, Phys. Rev. D 96 (2017) 024062 [arXiv:1703.06433] [INSPIRE].

[37] D. Bini, T. Damour and A. Geralico, Novel approach to binary dynamics: application to the fifth post-Newtonian level, Phys. Rev. Lett. 123 (2019) 231104 [arXiv:1909.02375] [INSPIRE].

[38] D. Bini, T. Damour and A. Geralico, Sixth post-Newtonian local-in-time dynamics of binary systems, Phys. Rev. D 102 (2020) 024061 [arXiv:2004.05407] [INSPIRE].

[39] D. Bini, T. Damour and A. Geralico, Sixth post-Newtonian nonlocal-in-time dynamics of binary systems, Phys. Rev. D 102 (2020) 084047 [arXiv: 2007.11239] [INSPIRE].

[40] D. Bini, T. Damour, A. Geralico, S. Laporta and P. Mastrolia, Gravitational dynamics at $O\left(G^{6}\right)$ : perturbative gravitational scattering meets experimental mathematics, arXiv: 2008.09389 [INSPIRE].

[41] G. Kälin and R.A. Porto, From Boundary Data to Bound States, JHEP 01 (2020) 072 [arXiv: 1910.03008] [INSPIRE].

[42] G. Kälin and R.A. Porto, From boundary data to bound states. Part II. Scattering angle to dynamical invariants (with twist), JHEP 02 (2020) 120 [arXiv: 1911.09130] [INSPIRE].

[43] G. Kälin, Z. Liu and R.A. Porto, Conservative Dynamics of Binary Systems to Third Post-Minkowskian Order from the Effective Field Theory Approach, Phys. Rev. Lett. 125 (2020) 261103 [arXiv:2007. 04977] [INSPIRE].

[44] J. Vines, J. Steinhoff and A. Buonanno, Spinning-black-hole scattering and the test-black-hole limit at second post-Minkowskian order, Phys. Rev. D 99 (2019) 064054 [arXiv:1812.00956] [INSPIRE].

[45] S. Foffa and R. Sturani, Effective field theory calculation of conservative binary dynamics at third post-Newtonian order, Phys. Rev. D 84 (2011) 044031 [arXiv:1104.1122] [INSPIRE].

[46] S. Foffa and R. Sturani, Effective field theory methods to model compact binaries, Class. Quant. Grav. 31 (2014) 043001 [arXiv:1309.3474] [INSPIRE].

[47] L. Blanchet and T. Damour, Postnewtonian Generation of Gravitational Waves, Ann. Inst. H. Poincare Phys. Theor. 50 (1989) 377 [InSPIRE].

[48] B. Kol and M. Smolkin, Non-Relativistic Gravitation: From Newton to Einstein and Back, Class. Quant. Grav. 25 (2008) 145011 [arXiv:0712.4116] [InSPIRE].

[49] B. Kol and M. Smolkin, Classical Effective Field Theory and Caged Black Holes, Phys. Rev. D 77 (2008) 064033 [arXiv:0712.2822] [INSPIRE].

[50] J.B. Gilmore and A. Ross, Effective field theory calculation of second post-Newtonian binary dynamics, Phys. Rev. D 78 (2008) 124021 [arXiv:0810.1328] [InSPIRE].

[51] J. Blümlein, A. Maier, P. Marquard and G. Schäfer, The fifth-order post-Newtonian Hamiltonian dynamics of two-body systems from an effective field theory approach: potential contributions, arXiv:2010.13672 [INSPIRE]. 